\title{
Knowledge of cervical cancer and attendance at cervical cancer screening: a survey of Black women in London
}

\author{
Christine Ekechi ${ }^{1}$, Adeola Olaitan ${ }^{2}$, Rosie Ellis ${ }^{3}$, Jacob Koris ${ }^{4}$, Adaugo Amajuoyi ${ }^{3}$ and Laura AV Marlow ${ }^{5^{*}}$
}

\begin{abstract}
Background: Women from ethnic minority backgrounds are less likely to attend cervical screening, but further understanding of ethnic inequalities in cervical screening uptake is yet to be established. This study aimed to explore the socio-demographic and ethnicity-related predictors of cervical cancer knowledge, cervical screening attendance and reasons for non-attendance among Black women in London.

Methods: A questionnaire was completed by women attending Black and ethnic hair and beauty specialists in London between February and April 2013. A stratified sampling frame was used to identify Black hair specialists in London subdivisions with $>10 \%$ Black population (including UK and foreign-born). Fifty-nine salons participated. Knowledge of cervical cancer risk factors and symptoms, self-reported screening attendance and reasons for non-attendance at cervical screening were assessed.

Results: Questionnaires were completed by 937 Black women aged 18-78, describing themselves as being predominantly from African or Caribbean backgrounds (response rate 26.5\%). Higher educational qualifications $(p<.001)$ and being born in the UK $(p=.011)$ were associated with greater risk factor knowledge. Older age was associated with greater symptom knowledge $(p<.001)$. Being younger, single, African (compared to Caribbean) and attending religious services more frequently were associated with being overdue for screening. Women who had migrated to the UK more than 10 years ago were less likely to be overdue than those born in the UK. Of those overdue for screening who endorsed a barrier (67/133), 'I meant to go but didn't get round to it' (28\%), fear of the test procedure (18\%) and low risk perception (18\%) were the most common barriers.

Conclusions: Ethnicity, migration and religiosity play a role in predicting cervical screening attendance among women from Black backgrounds. African women, those born in the UK and those who regularly attend church are most likely to put off attending. Additional research is needed to explore the attitudes, experiences and beliefs that explain why these groups might differ.
\end{abstract}

Keywords: Cervical screening, Knowledge, Awareness, Race, Ethnicity

\section{Background}

Cervical cancer is the fourth most common cancer in women worldwide with a significant burden of mortality, particularly in developing countries [1]. In developed countries such as the US and UK incidence of cervical cancer is much lower, but disparities by socio-economic status, race and ethnicity remain [2-5]. These disparities

\footnotetext{
*Correspondence: I.marlow@ucl.ac.uk

${ }^{5}$ Cancer Research UK Health Behaviour Research Centre, Department of Epidemiology \& Public Health, University College London, Gower Street, London WC1E 6BT, England

Full list of author information is available at the end of the article
}

are likely due to variation in risk factors for cervical cancer which include exposure to human papillomavirus, smoking status and non-attendance at cervical screening [6]. In England, an organised cervical screening programme dramatically reduces risk of cervical cancer, yet around $20 \%$ of women are not considered to be adequately screened [7].

A recent study that assessed a range of socio-economic variables, showed ethnicity was the most important sociodemographic predictor of having never attended cervical screening, with non-white women more than twice as 
likely to have never attended [8]. The term ethnicity refers to "the group to which people belong... as a result of certain shared characteristics including geographical and ancestral origins, but particularly cultural traditions and languages" [9]. A multi-faceted concept, ethnicity encompasses a range of factors such as migration history, language and religion. To date the influence that these factors have on cervical screening uptake has not been explored. Qualitative studies suggest lower knowledge of cervical cancer among minority groups could explain low engagement with cancer prevention services, [10] but this has not been explored quantitatively.

Identifying ethnic groups with poor knowledge of cervical cancer and low engagement with cancer prevention services would highlight where interventions to reduce inequalities need to be directed. A number of studies have examined factors associated with screening uptake in British South Asian women, [11] but to our knowledge there are no studies exploring cervical cancer knowledge or screening behaviour among Black women in the UK. In 2011, the Black population in England was approximately 1.8 million, including predominantly those from African and Caribbean backgrounds (UK-born and foreign-born) [12]. Women from African and Caribbean backgrounds are often grouped together for research purposes, but there is evidence of variation between these ethnic subgroups in other behavioural risk factors for cervical cancer (smoking status and sexual behaviour [13,14]). Differences in cervical screening uptake have not been explored.

We conducted a community-based survey of Black women in London. Our objectives were i) to explore socio-demographic and ethnicity-related factors associated with cervical cancer knowledge and non-attendance at cervical screening and ii) consider self-reported reasons for non-attendance at screening.

\section{Methods}

A questionnaire, examining knowledge and attitudes to cervical cancer screening was distributed to Black and ethnic hair and beauty specialists in London. We decided to focus data collection in London because census data (2011) show that more than half of England's Black population reside in London [12]. We planned to include all women over 18 years old, UK-born and foreign-born who classified themselves as African, Caribbean, any other Black/African/Caribbean background, mixed White and Black Carribbean, mixed White and Black African (in response to the 2011 census question on ethnic group). We felt that recruiting through hair and beauty salons serving the Black community would help us identify women from our target population including those who may not routinely access healthcare. Similar methods have been used for community-based recruitment of ethnic minority groups in the United States [15].

\section{Recruitment}

The aim was to distribute 6000 questionnaires through 100 establishments over a twelve week period. Initially, a stratified sampling frame was developed as follows, 1) London boroughs $s^{a}$ with a greater than $10 \%$ proportion of residents from Black backgrounds (including UK and foreign born) were selected, 2) The number of salons to be recruited within each borough was determined according to the size of the Black population in that borough, 3) A list of hair and beauty salons catering to the Black community within each of the selected boroughs were identified using an internet search (122 salons were identified). We intended to target 100 Salons across 18 boroughs, between 3 and 10 salons per borough (see Additional file 1). Where listed establishments had moved location or were unwilling to participate in the study, alternative local salons within the same borough were approached. Many of the salons on the original internet-based recruitment list were unavailable so a secondary recruitment method was based on identifying local high streets (focal points for business, particularly shops) in each borough and visiting these to look for salons. In some instances hair salons who agreed to participate would suggest other salons that might be interested in taking part. For logistical reasons, recruitment ceased after the allocated recruitment period (12 weeks) despite not reaching our original target of 100 salons.

Questionnaires were distributed between February and April 2013. Each participating salon was visited (by RE and/or AA) and left with 60 self-administered questionnaires to distribute to all women who attended over a one week period (7 days inclusive). Salons were given key rings to offer their clients as a thank you for participating. Some hair salons were visited on more than one occasion if at first visit it was apparent that the initial response rate was low. Questionnaires were completed anonymously, were sealed in an envelope provided and deposited in a secure box.

All women over the age of 18 who described themselves as being from a Black background were eligible for the study. Although women are not invited to cervical screening in England until they are 25 years old, we felt it was appropriate to assess knowledge of cervical cancer among women in a wider age range.

\section{Measures}

A self-administered structured questionnaire was developed based on previous research on cervical cancer knowledge and screening (Additional file 2). Socio-demographic and ethnicity-related variables were assessed using items from the 2001 Census. We assessed ethnicity, country of birth, length of time in the UK, language spoken at home, religion and religiosity. We also assessed education level, marital status and smoking status. Education was recoded into low (no formal qualifications or General Certificate of 
Secondary Education), medium (vocational qualifications or A-levels) or high (a university degree).

Knowledge questions were taken from the Cervical Cancer Awareness Measure (Cervical CAM) a validated tool to measure awareness of cervical cancer [16]. Two open questions from the Cervical CAM were used to examine knowledge of cervical cancer risk factors and symptoms. Participants freely listed risk factors and symptoms, and their responses were coded in accordance with a predefined list of target risk-factors and symptoms identified in the scientific literature and forming the National Health Service (NHS) Department of Health's 'Key Messages' on cervical cancer. Only correct risk factors and symptoms were coded.

To assess attendance at cervical screening women were asked "Which of these statements, if any, describes whether you have had cervical screening (smears)?" with the following response options: I have had a test within the last 3 years; My last test was 3 to 5 years ago; My last test was more than 5 years ago; I have never been invited to have a test; I have been invited but have never had a test; I have had a hysterectomy so I don't need to have tests; I have never heard of cervical screening; I am too young to be invited (18-24 years); None of the above. Women who had never had a smear test, or did not attend their last screening were asked to indicate why by selecting from a predefined list. The cervical screening attendance question and the predefined list of barriers had been used previously in a populationrepresentative survey [17].

\section{Analysis}

Data were analysed using SPSS v.20. Overall risk factor knowledge and symptom knowledge scores were calculated by allocating a score of ' 1 ' for each cited item that corresponded with the pre-defined list. Participants who did not cite any items on this list were allocated a score of ' 0 '. The same method was used in a previous study [18]. We have reported the means for these scores, but because the data were highly skewed participants were recoded into two groups, those who 'cited' vs 'did not cite' at least 1 correct risk factor/symptom. We used logistic regression to explore associations between each socio-demographic/ethnicityrelated variable and citing a correct risk factor/symptom. Logistic regression was also used to explore predictors of being overdue for screening. Women were considered 'overdue' for screening if they were 25-49 years and had not been screened in the last 3 years or if they were 49-64 and had not been screened in the last 5 years. Odds ratios $(\mathrm{OR})$ and $95 \%$ confidence intervals $(\mathrm{CI})$ are reported.

\section{Results}

A total of 3540 questionnaires were distributed through 59 Salons and 937 were returned (response rate - 26.5\%).
Blank questionnaires were excluded $(n=40)$, as were those with no completed demographic information provided $(n=21)$. Missing demographic data for the remaining 876 women was approximately $5 \%$ for most variables, but slightly higher for birthplace and migration year ( $8 \%$ and 10\%). Participants were only excluded from analyses involving variables where their data was missing.

\section{Sample characteristics}

Sample characteristics are shown in Table 1. Over half the women described themselves as Caribbean (56\%) and a quarter as African (25\%), with the rest describing their background as mixed White and Black Caribbean (5\%), mixed White and Black African (3\%) or other Black (4\%). Most spoke English as their main language (97\%). Most were born in the UK (61\%), but many of these women had a least one parent born outside the UK (84\%). A third of women were born outside of the UK, but most of these women had been living in the UK for more than 10 years. The majority were Christian (77\%), with $41 \%$ attending religious service at least once a month. Almost half were single (48\%) and 50\% had a high level of educational qualifications.

\section{Knowledge of cervical cancer symptoms and risk factors}

Just over half of women cited at least one cervical cancer risk factor $(55 \%, \mathrm{n}=481)$ and $58 \%(\mathrm{n}=509)$ cited at least one cervical cancer symptom. While $41 \%$ of women cited both a risk factor and a symptom, $28 \%$ could not cite either. The percentage of women citing each risk factor/symptom is shown in Table 2.

The most commonly cited risk factors were, having many sexual partners (15\%), smoking (12\%) and not going for smear tests (10\%). Only $4 \%$ cited infection with human papillomavirus, but $29 \%$ cited at least one risk factor relating to sexual behaviour (having multiple sexual partners, sex at a young age, unprotected sex, HPV or other STIs). On average women cited 0.61 correct risk factors (range 0-6). Citing at least one correct risk factor was not associated with ethnicity, length of time in the UK, religion or religiosity, however women who were born in the UK were more likely to cite a risk factor than women born outside the UK $(\mathrm{OR}=1.46, \mathrm{CI}$ : 1.09-1.96). Women with a high level of education were more likely to cite a risk factor than those with a low level of education $(\mathrm{OR}=2.22, \mathrm{CI}$ : 1.46-3.35). Age and marital status were not significantly associated with risk factor knowledge. In adjusted analyses, including education and being born in the UK, both variables remained significant.

The most commonly cited symptoms were vaginal bleeding in-between periods and unusual vaginal discharge (cited by $32 \%$ and $25 \%$ of women respectively). 
Table 1 Sample characteristics

\begin{tabular}{|c|c|c|c|c|}
\hline & \multicolumn{2}{|c|}{ Whole sample $(n=876)$} & \multicolumn{2}{|c|}{ Screen eligible $(n=652)$} \\
\hline & $\bar{N}$ & \%* & $\bar{N}$ & \%* \\
\hline \multicolumn{5}{|l|}{ Marital status } \\
\hline Single & 417 & 47.6 & 295 & 45.1 \\
\hline Married & 214 & 24.4 & 184 & 28.4 \\
\hline Cohabiting & 95 & 10.8 & 77 & 11.8 \\
\hline Divorced/separated/widowed & 106 & 12.1 & 94 & 14.4 \\
\hline \multicolumn{5}{|l|}{ Education } \\
\hline Low level & 131 & 15.0 & 100 & 15.3 \\
\hline Medium level & 240 & 27.4 & 162 & 24.8 \\
\hline High level & 436 & 49.8 & 348 & 53.4 \\
\hline Other & 30 & 3.4 & 25 & 3.8 \\
\hline \multicolumn{5}{|l|}{ Ethnicity } \\
\hline Caribbean & 488 & 55.7 & 380 & 58.3 \\
\hline African & 216 & 24.7 & 166 & 25.5 \\
\hline Mixed white and Black Caribbean & 39 & 4.5 & 33 & 5.1 \\
\hline Mixed white and Black African & 25 & 2.9 & 20 & 3.1 \\
\hline Any other Black background & 33 & 3.8 & 28 & 4.3 \\
\hline Other mixed background & 13 & 1.5 & 9 & 1.4 \\
\hline Other & 18 & 2.1 & 11 & 1.7 \\
\hline \multicolumn{5}{|l|}{ Birthplace } \\
\hline UK-born and parents UK-born & 83 & 9.5 & 46 & 7.1 \\
\hline UK-born, 1 parent UK-born & 204 & 23.3 & 159 & 24.4 \\
\hline UK born, no parents UK-born & 244 & 27.9 & 205 & 31.4 \\
\hline Not UK-born & 275 & 31.4 & 221 & 33.9 \\
\hline \multicolumn{5}{|l|}{ Year moved to UK } \\
\hline 1956-1972 & 49 & 5.5 & 35 & 5.4 \\
\hline 1973-1990 & 69 & 7.9 & 62 & 9.5 \\
\hline 1991-2002 & 95 & 10.8 & 77 & 11.8 \\
\hline $2003-2013$ & 44 & 5.0 & 34 & 5.2 \\
\hline \multicolumn{5}{|l|}{ Religion } \\
\hline Christian & 678 & 77.4 & 498 & 76.4 \\
\hline Muslim & 27 & 3.1 & 23 & 3.5 \\
\hline No religion & 103 & 11.8 & 84 & 12.9 \\
\hline Other & 27 & 3.1 & 25 & 3.8 \\
\hline \multicolumn{5}{|l|}{ Religious service attendance } \\
\hline Rarely or never & 238 & 27.2 & 181 & 27.8 \\
\hline Few times a year & 239 & 27.3 & 180 & 27.6 \\
\hline 1-3 times a month & 103 & 11.8 & 75 & 11.5 \\
\hline At least once a week & 251 & 28.7 & 192 & 29.4 \\
\hline \multicolumn{5}{|l|}{ Smoking Status } \\
\hline I have never smoked & 508 & 58.0 & 379 & 58.1 \\
\hline I used to smoke & 210 & 24.0 & 162 & 24.8 \\
\hline I currently smoke & 137 & 15.6 & 101 & 15.5 \\
\hline
\end{tabular}

*Unaccounted percentage is missing data. 


\begin{tabular}{lll}
\multicolumn{4}{l}{ Table $\mathbf{2}$ Cervical cancer risk factor and symptoms } \\
knowledge (n = 876) & N & \%* \\
\hline & & \\
\hline Risk factors for cervical cancer & 132 & 15.1 \\
Having many sexual partners & 102 & 11.6 \\
Smoking & 89 & 10.2 \\
Not going for regular smear tests & 57 & 6.5 \\
Unprotected sex & 48 & 5.5 \\
Infection, STI/STD or virus & 44 & 5.0 \\
Starting to have sex at a young age & 38 & 4.3 \\
Infection with Human papillomavirus & 17 & 1.9 \\
Long term use of the contraceptive pill & 5 & 0.6 \\
Having many children & 3 & 0.3 \\
Having a weakened immune system & & \\
Symptoms of cervical cancer & 282 & 32.2 \\
Vaginal bleeding between periods & 219 & 25.0 \\
Abnormal discharge & 145 & 16.6 \\
Persistent pelvic/abdominal pain & 78 & 8.9 \\
Pain/discomfort during sex & 56 & 6.4 \\
Vaginal bleeding during/after sex & 13 & 1.5 \\
Persistant lower back pain & 13 & 1.5 \\
Blood in stool/urine & 4 & 1.4 \\
Unexplained weight loss & 0.7 \\
Vaginal bleeding after the menopause & 0.5 \\
\hline
\end{tabular}

*Percentage of entire sample; Women were able to select more than one risk factor/symptom.

Note: Risk factors/Symptoms identified in the NHS Department of Health's 'Key Messages' on cervical cancer [18].

Less than $1 \%$ of women cited heavier/longer periods or vaginal bleeding after the menopause. On average women cited 0.95 symptoms (range 0-5). Age was the only variable significantly associated with citing at least one correct symptom $(\mathrm{p}<.001)$. Compared to $16-24$ year olds each older age group was progressively more likely to cite a correct symptom: $25-34$ years $(\mathrm{OR}=$ 2.54, CI: $1.55-4.18)$, 35-44 years $(\mathrm{OR}=2.76, \mathrm{CI}: 1.66$ $4.57)$, 45-64 years $(\mathrm{OR}=4.52$, CI: 2.73-7.49). Women over 65 years were no more likely to cite a correct symptom than the youngest group $(p>.05)$. Symptom knowledge was not associated with ethnicity, being born in the UK, religion or religiosity or education. Marital status was associated with citing a correct symptom, but this was no longer the case when adjusting for age.

\section{Attendance at screening}

Of the eligible population (those aged 25-64 years, who had not had a hysterectomy, $\mathrm{n}=652$ ), 75\% had been screened in the last 3 years and $16 \%$ in the last $3-5$ years.
Sample characteristics for the screen eligible women are shown in Table 1. Most women were last screened at their GP surgery (75\%), but health centres (10\%), hospitals (7\%) and private clinics (2\%) were used by some women. Overall, $20.4 \%$ of women were overdue for screening $(\mathrm{n}=133)$. In univariable analyses (see Table 3 ) women were more likely to be overdue screening if they were younger (23\% of $25-34$ year olds and $25 \%$ of $35-44$ year olds compared to $13 \%$ of $45-64$ year olds, $\mathrm{p}=.007$ and .003 respectively) and single (27\% compared with $15 \%$ of married/cohabiting women, $\mathrm{p}=.001)$. Women who considered themselves to be African were more likely to be overdue ( $26 \%$ compared with $18 \%$ of those who considered themselves to be Caribbean, $\mathrm{p}=.024)$. Women who had migrated to the UK more than 10 years ago were less likely to be overdue for screening than women born in the UK $(12 \%$ compared with $23 \%, \mathrm{p}=.003)$. Women who attended religious services at least once a week were more likely to be overdue than those who rarely or never attended (27\% compared with $17 \%, \mathrm{p}=.020)$. Education level and religion were not significantly associated with screening status. Women who cited at least one symptom were less likely to be overdue for screening $(\mathrm{OR}=0.58, \mathrm{CI}$ : 0.40-0.85). In a multivariable model all variables remained significant except for ethnicity and symptom knowledge (see Table 3 ).

\section{Reasons for non-attendance in those overdue for screening}

Of the 133 women who were overdue for screening, half selected at least one reason for not attending from the list provided $(n=67$, see Table 4$)$. Younger women $\left(x^{2}\right.$ $(2)=6.54, p=.038)$, single women $\left(\chi^{2}(2)=8.05, p=.018\right)$ and those with the highest level of education $\left(\chi^{2}(2)=\right.$ 12.06, $\mathrm{p}=.002$ ) were more likely to provide reasons for non-attendance, but there were no differences by ethnicity, birthplace, or religiosity. Of those who selected a reason for never being screened or delaying screening, 'I meant to go but didn't get round to it' (28\%), fear of the test procedure (18\%) and low risk perception (18\%) were the most common responses. Table 4 shows the reasons women endorsed by ethnicity, birthplace and religiosity, but numbers were too small to analyse this statistically.

\section{Discussion}

We explored socio-demographic predictors of cervical cancer knowledge and cervical screening behaviour among Black women living in London.

For cervical cancer knowledge, education level was the most important predictor of citing a risk factor, while age was the most important predictor of citing a symptom, consistent with other studies in the cervical cancer context $[18,19]$. To the most part, none of the ethnicityrelated variables were associated with knowledge, with 
Table 3 Associations between socio-demographic variables and being overdue for screening

\begin{tabular}{|c|c|c|c|}
\hline & & Univariable analyses & Multivariable analyses ${ }^{1}$ \\
\hline & Overdue n (\%) & OR $[95 \% \mathrm{Cl}]$ & OR $[95 \% \mathrm{Cl}]$ \\
\hline \multicolumn{4}{|l|}{ Age Group } \\
\hline $25-34$ & $54(23.3)$ & $1.98[1.21-3.25]^{* *}$ & $1.35[0.75-2.42]$ \\
\hline $35-44$ & $50(24.9)$ & $2.16[1.32-3.58]^{* *}$ & $1.92[1.09-3.37]^{*}$ \\
\hline $45-64$ & $29(13.3)$ & 1.00 & \\
\hline \multicolumn{4}{|l|}{ Marital Status } \\
\hline Single & 79 (26.9) & $2.03[1.33-3.10]^{* *}$ & $2.11[1.32-3.37]^{* *}$ \\
\hline Married/Cohabiting & $40(15.3)$ & 1.00 & \\
\hline Divorced/separated/widowed & $81(13.8)$ & $0.89[0.45-1.74]$ & $0.98[0.45-2.14]$ \\
\hline \multicolumn{4}{|l|}{ Education } \\
\hline Low level & $15(15.0)$ & 0.67 & \\
\hline Medium level & $37(22.8)$ & $1.13[0.37-1.24]$ & \\
\hline High level & $72(20.7)$ & $1.00[0.72-1.77]$ & \\
\hline \multicolumn{4}{|l|}{ Ethnicity } \\
\hline Caribbean & $74(17.9)$ & 1.00 & \\
\hline African & $48(25.9)$ & $1.61[1.06-2.44]^{*}$ & $1.59[0.97-2.60]$ \\
\hline Other & $9(19.6)$ & $1.12[0.52-2.42]$ & $1.29[0.57-2.92]$ \\
\hline \multicolumn{4}{|l|}{ Birthplace } \\
\hline Born in the UK & $95(23.2)$ & 1.00 & \\
\hline Migrated <10 years ago & $11(32.4)$ & $1.59[0.75-3.37]$ & $1.01[0.44-2.29]$ \\
\hline Migrated $>10$ years ago & $21(12.1)$ & $0.46[0.28-0.76]^{* *}$ & $0.42[0.24-0.75]^{* *}$ \\
\hline \multicolumn{4}{|l|}{ Religion } \\
\hline Christian & $109(21.9)$ & 1.00 & \\
\hline Muslim & $6(26.1)$ & $1.26[0.48-3.26]$ & \\
\hline No religion & $11(13.1)$ & $0.54[.275-1.05]$ & \\
\hline \multicolumn{4}{|l|}{ Religious service attendance } \\
\hline Rarely or never & $60(16.6)$ & 1.00 & \\
\hline Few times a year & $36(20.0)$ & $1.26[0.74-2.15]$ & $1.27[0.72-2.26]$ \\
\hline 1-3 times a month & $11(14.9)$ & $0.88[0.42-1.86]$ & $1.04[0.48-2.28]$ \\
\hline At least once a week & $51(26.6)$ & $1.82[1.10-3.02]^{*}$ & $2.05[1.16-3.54]^{*}$ \\
\hline \multicolumn{4}{|l|}{ Risk factor knowledge score } \\
\hline 0 & $78(22.5)$ & 1.00 & \\
\hline $1+$ & $55(18.0)$ & $0.76[0.51-1.11]$ & \\
\hline \multicolumn{4}{|l|}{ Symptom knowledge score } \\
\hline 0 & $68(25.8)$ & 1.00 & \\
\hline $1+$ & $65(16.8)$ & $0.58[0.40-0.85]^{* *}$ & $0.72[0.47-1.09]$ \\
\hline
\end{tabular}

${ }^{1}$ Includes: age group, marital status, ethnicity, birthplace, religious service attendance, Symptom knowledge score. ${ }^{*} \mathrm{p}<.05 ;{ }^{* *} \mathrm{p}<.01$.

the exception being migration status. In general, the most commonly reported risk factors (many sexual partners and smoking) and symptoms (unusual vaginal bleeding and persistent unusual discharge) in our study and the population-representative study were the same [18]. Interestingly, persistent pain was more commonly cited as a symptom in our study (17\% compared with
$1 \%$ ) and this could be a cause for concern if it means symptoms in the absence of pain are not taken seriously by women from Black backgrounds. Previous work in the context of breast cancer suggests some African American women feel confused over whether pain is a symptom of cancer [20] and this warrants further investigation. 
Table 4 Reasons for non-attendance among women overdue for screening (number endorsed, per cent in brackets)

\begin{tabular}{|c|c|c|c|c|c|c|c|c|}
\hline & \multirow[b]{2}{*}{$\begin{array}{l}\text { Overall } \\
(n=67)\end{array}$} & \multicolumn{2}{|c|}{ Ethnicity } & \multicolumn{2}{|c|}{ Birthplace } & \multicolumn{3}{|c|}{ Religiosity* } \\
\hline & & $\begin{array}{c}\text { Caribbean } \\
(n=35)\end{array}$ & $\begin{array}{l}\text { African } \\
(n=29)\end{array}$ & $\begin{array}{l}\text { UK born } \\
(n=20)\end{array}$ & $\begin{array}{c}\text { Not UK born } \\
(n=47)\end{array}$ & $\begin{array}{c}\text { None/low } \\
(n=18)\end{array}$ & $\begin{array}{c}\text { Medium } \\
(n=21)\end{array}$ & $\begin{array}{c}\text { High } \\
(n=27)\end{array}$ \\
\hline \multicolumn{9}{|l|}{ Practical barriers } \\
\hline I meant to go but didn't get around to it & $19(28.4)$ & $11(31.4)$ & $8(27.6)$ & $11(23.4)$ & $8(40.0)$ & $6(33.3)$ & $5(23.8)$ & $7(25.9)$ \\
\hline It is difficult to get an appointment at a time that suits me & $11(16.4)$ & $9(25.7)$ & $1(3.4)$ & $11(23.4)$ & $0(0)$ & $3(16.7)$ & $4(19.0)$ & $4(14.8)$ \\
\hline Too busy/inconvenient & $9(13.4)$ & $5(14.3)$ & $4(13.8)$ & $5(10.6)$ & $4(20.0)$ & $4(22.2)$ & $1(4.8)$ & $4(14.8)$ \\
\hline \multicolumn{9}{|l|}{ Emotional Barriers } \\
\hline Fear of the test procedure & $12(17.9)$ & $3(8.6)$ & $9(31.0)$ & $9(19.1)$ & $3(15.0)$ & $1(5.6)$ & $5(23.8)$ & $6(22.2)$ \\
\hline Fear of a 'bad' result & $10(14.9)$ & $5(14.3)$ & $5(17.2)$ & $6(12.8)$ & $4(20.0)$ & $3(16.7)$ & $4(19.0)$ & $3(11.1)$ \\
\hline Bad experience of cervical screening in the past & $6(9.0)$ & $3(8.6)$ & $3(10.3)$ & $6(12.8)$ & $0(0)$ & $4(22.2)$ & $1(4.8)$ & $1(3.7)$ \\
\hline Embarrassment & $6(9.0)$ & $2(5.7)$ & $4(13.8)$ & $5(10.6)$ & $1(5.0)$ & $1(5.6)$ & $2(9.5)$ & $3(11.1)$ \\
\hline \multicolumn{9}{|l|}{ Beliefs about screening } \\
\hline I do not believe I am at risk & $12(17.9)$ & $4(11.4)$ & $7(24.1)$ & $9(19.1)$ & $3(15.0)$ & $5(27.8)$ & $1(4.8)$ & $6(22.2)$ \\
\hline I do not believe the test is needed & $4(6.0)$ & $0(0)$ & $3(10.3)$ & $2(4.3)$ & $2(10.0)$ & $2(11.1)$ & $1(4.8)$ & $1(3.7)$ \\
\hline \multicolumn{9}{|l|}{ Other } \\
\hline I have never been invited for screening & $9(13.4)$ & $4(11.4)$ & $5(17.2)$ & $5(10.6)$ & $4(20.0)$ & $1(5.6)$ & $3(14.3)$ & $5(18.5)$ \\
\hline I didn't understand the screening invitation letter & $1(1.5)$ & $1(2.9)$ & 0 & $1(2.1)$ & $0(0)$ & $1(5.6)$ & $0(0)$ & $0(0)$ \\
\hline
\end{tabular}

*None/low: Rarely or never; Medium: 1-3 times a month/A few times a year; High: At least once a month.

We also explored non-attendance at cervical screening. Younger age and being single were associated with being overdue for screening, consistent with other studies $[17,21,22]$. Many of the ethnicity-related variables were also associated with being overdue for screening. The percentage of those who were overdue for cervical screening was higher in those who had recently migrated to the UK (32\%), but much lower in those who had been in the UK for more than 10 years (12\%). This is consistent with studies in the US that have shown immigrants are less likely to have been screened but this inequality decreases with acculturation (length of time in the new country) $[23,24]$. There were also differences between subgroups of the Black population, with women from African backgrounds more likely to be overdue for screening than Caribbean women. This is interesting when considered in the context of other cervical cancer risk factors (smoking and having multiple sexual partners) which suggest that African women may be at lower risk of cervical cancer than Caribbean women $[13,14]$. Agyemang et al. [25] argued that health researchers should consider diversity within Black populations because differences in beliefs and behaviours exist, yet there remains a paucity of research exploring differences in cancer beliefs between African and Caribbean women in the UK.

Non-attenders reported not getting round to screening, difficulty making an appointment, fear of the test procedure and fear of a bad result as barriers to screening. African women who were overdue screening were more likely to endorse fear of the test and embarrassment and to believe they were not at risk of cervical cancer. Small numbers mean we could not run statistical analyses on this data so this needs further exploration. Similar to other studies with Black women we found an association between symptom knowledge and screening behaviour [26] which could suggest interventions designed to increase awareness might consequently have a positive influence on behaviour. Alternatively, cervical screening could be acting as an opportunity for women to learn about cervical cancer risk factors and symptoms.

\section{Strengths and limitations}

To our knowledge this is the first study to explore cervical cancer knowledge and screening behaviour with a focus on Black women living in London. We took a novel approach to recruitment, targeting a specific ethnic group by approaching women in the community. We felt that targeting women through hairdressers, a non-health care setting would help us recruit those who do not always engage with health care. Using this method, we successfully recruited a large number of women with a good range on the ethnicity-related variables. We used questions from validated measures used in previous population-based surveys, although we did not pilot our survey before implementing the study.

This study has some limitations. The response rate was low $(26.5 \%)$, so we cannot assume our findings are generalisable to the entire population of women from Black ethnic backgrounds living in London. In comparison to 2011 
census statistics for the London boroughs we sampled from, we recruited more Black Caribbean women $(58 \%$ vs $29 \%$ ) and fewer African women (25\% vs $45 \%$ ), with fewer women born outside the UK ( $31 \%$ vs $46 \%$ - figure for women of all ages). One reason for this could be that the survey was completed in written English, thereby excluding women who cannot read English, who are more likely to born outside of the UK and be from African backgrounds. Around half of our sample had degreelevel qualifications and this is also higher than population-level figures: $40 \%$ for men and women from Black African and 26\% from Black Caribbean backgrounds. Five-yearly screening uptake in our study was $91 \%$, which is higher than the national figure $(80 \%)$ and we acknowledge that this suggests non-attenders were under-represented. However, more women were overdue for screening in this study than in a recent population-representative survey which used 'goldstandard' recruitment methods [17]. It is also possible that by relying on self-reported screening attendance our findings are subject to social desirability bias, despite our attempts to minimise this by ensuring anonymity. These limitations have important implications and suggest that the overall percentages we report should be interpreted with caution.

Our decision to focus recruitment in London means we cannot necessarily extrapolate our findings to Black communities in other parts of the UK, both rural and urban. Recruiting through hair salons means women who do not attend salons frequently or at all, perhaps the more socially isolated or those from lower socio-economic backgrounds, are less likely to be included. We also relied on salon staff to distribute and collect the questionnaire (the denominator for the response rate is the number of questionnaires distributed to salons rather than the number of women approached). Difficulties with identifying and engaging salons meant we had to adapt our methodology and ultimately did not reach our initial recruitment target. We feel our experience may have important implications for other researchers considering a similar communitybased approach to survey recruitment and have provided more detail about our experience (see Additional file 3).

\section{Conclusions}

This study suggests that ethnicity, migration and religiosity play a role in predicting cervical screening attendance among Black women in London. Although previous work had suggested ethnic inequalities in cervical screening uptake between white and non-white women, [8] this is the first study to suggest that among Black women those from African backgrounds, and those who attend religious service on a frequent basis could be the most likely to delay attending cervical screening. More research is needed to explore the attitudes, experiences and beliefs that might explain why these groups differ.

\section{Details of ethical approval}

The study was approved by the UCL research ethics committee (ref:0496/011).

\section{Endnote}

${ }^{a}$ Boroughs are subdivisions of greater London, each governed by a different council. London has 32 boroughs, each with a population of approximately 150,000-363,000 [27].

\section{Additional files}

\section{Additional file 1: Sampling frame.}

Additional file 2: Questionnaire: Attitudes to cervical screening among women attending specialist black hairdressers in London.

Additional file 3: Difficulties with recruitment.

\section{Competing interests}

The authors declare that they have no competing interests.

\section{Authors' contributions}

AO, LM and CE conceived of and designed the study. JK, RE and AA collected the data. LM analysed the data. CE and LM interpreted the data and drafted the manuscript. All authors contributed to critically revising the manuscript and approving the final version.

\section{Acknowledgements}

We would like to thank all the salons that participated for their help with the recruitment and Jo's Cervical Cancer Trust for contributing the trolley token key-rings which were used as an incentive. Remaining study costs were covered by the UCL charity account. LM is funded by Cancer Research UK. $\mathrm{CE}$ and $\mathrm{AO}$ are employed by the NHS. At the time of the study RE, JK and AA were medical students studying at UCLH. Funders played no role in the study.

\section{Funding}

The study was funded by the UCL charity account.

\section{Author details}

'Department of Obstetrics \& Gynaecology, Imperial College Healthcare Trust, London W12 OHS, England. 'University College London Hospital, 2nd floor North, 250 Euston Road, London NW1 2PG, England. ${ }^{3}$ University College London Medical School, 21 University Street, London WC1E 6DE, England. ${ }^{4}$ Leicester Royal Infirmary, Infirmary Square, Leicester LE1 5WW, England. ${ }^{5}$ Cancer Research UK Health Behaviour Research Centre, Department of Epidemiology \& Public Health, University College London, Gower Street, London WC1E 6BT, England.

Received: 19 June 2014 Accepted: 15 October 2014

Published: 22 October 2014

\section{References}

1. IARC: GLOBOCAN 2012: estimated cancer incidence, mortality abndf prevalence worldwide in 2012. 2012. http://globocan.iarc.fr/Pages/ fact_sheets_cancer.aspx.

2. CDC: Cervical cancer rates by race and ethncity. http://www.cdc.gov/ cancer/cervical/statistics/race.htm.

3. Shack $\mathrm{L}$, Jordan $\mathrm{C}$, Thomson CS, Mak V, Moller H: Variation in incidence of breast, lung and cervical cancer and malignant melanoma of skin by socioeconomic group in England. BMC Cancer 2008, 8:271.

4. NCIN: Cancer incidence and survival by major ethnic group, England, 2002-2006. http://publications.cancerresearchuk.org/downloads/product/ CS_REPORT_INCSURV_ETHNIC.pdf. 
5. Ward E, Jemal A, Cokkinides V, Singh GK, Cardinez C, Ghafoor A, Thum M: Cancer disparities by race/ethnicity and socioeconomic status. CA Cancer J Clin 2004, 54:78-93.

6. Currin LG, Jack RH, Linklater KM, Mak V, Moller H, Davies EA: Inequalities in the incidence of cervical cancer in South East England 2001-2005: an investigation of population risk factors. BMC Public Health 2009, 9:62

7. Screening and Immunisations team H\&SCIC: NHS Cervical Screening Programme Statistical Bulletin (England 2012-13). http://www. cancerscreening.nhs.uk/cervical/statistics-bulletin.html.

8. Moser K, Patnick J, Beral V: Inequalities in reported use of breast and cervical screening in Great Britain: analysis of cross sectional survey data. BMJ 2009, 338:b2025

9. Bhopal R: Glossary of terms relating to ethnicity and race: for reflection and debate. J Epidemiol Community Health 2004, 58:441-445.

10. Thomas VN, Saleem T, Abraham R: Barriers to effective uptake of cancer screening among Black and minority ethnic groups. Int J Palliat Nurs 2005, 11:564-571.

11. Sokal R: A critical review of the literature on the uptake of cervical and breast screening in British South Asian women. Qual Prim Care 2010, $18: 251-261$

12. Office for National Statistics: 2011 Census: KS201EW Ethnic group, local authorities in England and Wales. http://www.ons.gov.uk/ons/rel/census/ 2011-census/key-statistics-for-local-authorities-in-england-and-wales/rfttable-ks201ew.xls.

13. Sproston K, Mindell J: Health Survey for England 2004. The Health of Minority Ethnic Groups. London: The Information Centre; 2006

14. Fenton KA, Mercer CH, McManus S, Erens B, Wellings K, Macdowall W, Byron CL, Copas AJ, Nanchahal K, Field J, Johnson AM: Ethnic variations in sexual behaviour in Great Britain and risk of sexually transmitted infections: a probability survey. Lancet 2005, 365:1246-1255.

15. Guthrie LC, Butler SC, Ward MM: Time perspective and socioeconomic status: a link to socioeconomic disparities in health? Soc Sci Med 2009 68:2145-2151

16. Simon AE, Wardle J, Grimmett C, Power E, Corker E, Menon U, Matheson L, Waller J: Ovarian and cervical cancer awareness: development of two validated measurement tools. J Fam Plann Reprod Health Care 2012, 38:167-174.

17. Waller J, Bartoszek M, Marlow L, Wardle J: Barriers to cervical cancer screening attendance in England: a population-based survey. J Med Screen 2009, 16:199-204.

18. Low EL, Simon AE, Lyons J, Romney-Alexander D, Waller J: What do British women know about cervical cancer symptoms and risk factors? Eur J Cancer 2012, 48:3001-3008.

19. Waller J, McCaffery K, Wardle J: Beliefs about the risk factors for cervical cancer in a British population sample. Prev Med 2004, 38:745-753.

20. Jones CE, Maben J, Jack RH, Davies EA, Forbes LJ, Lucas G, Ream G: A systematic review of barriers to early presentation and diagnosis with breast cancer among black women. BMJ Open 2014, 4:e004076.

21. Marlow LA, Waller J, Wardle J: Sociodemographic predictors of HPV testing and vaccination acceptability: results from a populationrepresentative sample of British women. J Med Screen 2008, 15:91-96.

22. Lancucki L, Fender M, Koukari A, Lynge E, Mai V, Onysko J, Ronco G, Tornberg S, Vessey M, Patnick J: A fall-off in cervical screening coverage of younger women in developed countries. J Med Screen 2010, 17:91-96.

23. Swan J, Breen N, Coates RJ, Rimer BK, Lee NC: Progress in cancer screening practices in the United States: results from the 2000 National Health Interview Survey. Cancer 2003, 97:1528-1540.

24. Brown WM, Consedine NS, Magai C: Time spent in the United States and breast cancer screening behaviors among ethnically diverse immigrant women: evidence for acculturation? J Immigr Minor Health 2006, 8:347-358.

25. Agyemang C, Bhopal R, Bruijnzeels M: Negro, Black, Black African, African Caribbean, African American or what? Labelling African origin populations in the health arena in the 21st century. J Epidemio/ Community Health 2005, 59:1014-1018.
26. Bynum SA, Guillaume DA, Brandt HM, Fletcher FE: Does knowledge influence pap test screening among young African-American women? J Cancer Educ 2014, 29:478-481.

27. GLA Intelligence: 2011 Census first results: London boroughs' populations by age by sex. 2012, Update CIS2012-01. https://londondatastore-upload.s3. amazonaws.com/9i0\%3D2011-census-first-results.pdf.

doi:10.1186/1471-2458-14-1096

Cite this article as: Ekechi et al:: Knowledge of cervical cancer and attendance at cervical cancer screening: a survey of Black women in London. BMC Public Health 2014 14:1096.

\section{Submit your next manuscript to BioMed Central and take full advantage of:}

- Convenient online submission

- Thorough peer review

- No space constraints or color figure charges

- Immediate publication on acceptance

- Inclusion in PubMed, CAS, Scopus and Google Scholar

- Research which is freely available for redistribution

Submit your manuscript at www.biomedcentral.com/submit
C BioMed Central 